\title{
EVALUACIÓN EN POSTCOSECHA DE LISIANTHUS (EUSTOMA GRANDIFLORUM) C.V. HEIDI, DESTINADO COMO FLOR DE CORTE AL MERCADO LOCAL
}

\author{
POST-HARVEST EVALUATION OF LISIANTHUS (EUSTOMA GRANDIFLORUM) \\ C.V. HEIDI, DESTINED AS FLOWER TO THE LOCAL MARKET
}

\author{
Nelson Loyola López ${ }^{1}$; Sandra Guzmán Cornejo ${ }^{1}$
}

\begin{abstract}
RESUMEN
El rubro de la floricultura se ha convertido en los últimos años en una interesante opción productiva para el país. Sin embargo, los pequeños productores se ven excluidos de la posibilidad de exportar sus productos y poder así obtener mejores precios, debido a las precarias condiciones en que almacenan las flores una vez cortadas y a la falta de conocimientos sobre tratamientos de postcosecha adecuados, para cada especie floral.

Debido a esto, se hace necesaria la búsqueda de nuevas alternativas productivas, en las que se combinen flores con una buena vida de postcosecha y tratamientos de postcosecha que se encuentren al alcance de los pequeños productores.

Con la finalidad de aportar a esta búsqueda se realizó un ensayo con el propósito de evaluar el comportamiento de postcosecha de Lisianthus (Eustoma grandiflorum), utilizando siete tratamientos, en los cuales se utilizó el producto aplicado en precosecha Como® y ácido cítrico aplicado en postcosecha, evaluando cuatro parámetros de calidad: Número de flores abiertas, número de flores senescentes, diámetro del botón y peso del botón. El tratamiento $\mathrm{T}_{4}$, que correspondió a la aplicación de Como® en precosecha, con una concentración de $1 \mathrm{cc} / \mathrm{L}$, más $0,5 \mathrm{~mL}$ de ácido cítrico/L de agua en postcosecha, presentó efectos positivos en la vida de postcosecha de Lisianthus, optimizando el diámetro del botón floral y el peso de este. Con respecto al resto de los tratamientos, no se encontró diferencia significativa entre ellos.
\end{abstract}

Palabras claves: Lisianthus (Eustoma grandiflorum), apertura floral.

\begin{abstract}
In the recent years, the flower production has been an interesting productive option for Chile. However, the small farmers are excluded of the possibility to export their flowers as well as to obtain better prices, due to the precarious conditions in which they store and also the lack of knowledge of postharvest alternatives for each floral species.

In this way, it becomes necessary to study new production alternatives in those that combine flowers with a good postharvest treatment according to the economical conditions of the small farmers.

In relation to the contribution of this research, it was carried out an experiment which evaluated the behavior of Lisianthus (Eustoma grandiflorum R.) provinding storage conditions $\left(12{ }^{\circ} \mathrm{C} ; 85 \%\right.$ H.R.) by using seven treatments, in which the products were applied in preharvest (Como $\left.{ }^{\circledR}\right)$ and also citric acid applied in postharvest. In all of them there were evaluated four parameters of quality: Number of open flowers, number of flowers senecens, diameter of the button and weight of the button.

The treatment $T_{4}$ that corresponded to the application of Como® in preharvest, with a concentration of $1 \mathrm{cc} / \mathrm{L}$, plus $0,5 \mathrm{~mL}$ of citric acid/L of water in postharvest, exhibited positive effects in the shelf life of Lisianthus, optimizing the diameter of the floral button and its weight.
\end{abstract}

Key words: Lisianthus (Eustoma grandiflorum), floral opening.

1 Departamento de Ciencias Agrarias, Universidad Católica del Maule. E-mail: nloyola@ucm.cl-loyola@ctcinternet.cl. Fono: (75)-371140. Casilla 7-D Curicó, VII Región, Chile.

Fecha de Recepción: 16 Marzo 2007

Fecha de Aceptación: 20 Junio 2007 


\section{INTRODUCCIÓN}

Chile presenta condiciones climáticas y fitosanitarias muy favorables para la producción de flores, las que son comercializadas tanto en el mercado nacional como en el mercado externo, con lo que el rubro de la floricultura se ha convertido en una interesante opción productiva para la agricultura nacional.

El primer registro comercial data de fines de la década del 70, correspondiendo al cultivo del clavel, el cual provenía especialmente de la V Región y se destinaba al mercado nacional. Las primeras exportaciones fueron realizadas a partir del año 1978, con destino a Paraguay y Gran Bretaña, y paralelamente se desarrollaron los primeros invernaderos. Con el cultivo de nuevas especies, ya en los años 80, la floricultura comenzó lentamente su expansión. En los inicios del 90 empezaron a consolidarse algunas empresas, y a mediados de la década se incorporaron nuevas flores a las exportaciones, $\mathrm{y}$ se diversificó el destino de los envíos ${ }^{1}$.

En nuestro país, actualmente se producen alrededor de 20 especies, las cuales se orientan principalmente a flores de corte; clavel, lilium, tulipán, liatris, entre otras, como de producción de bulbos, lilium y tulipán y, en menor proporción, a la producción de semillas de flores. Según el Censo Nacional Agropecuario de 1997, la floricultura ocupa una superficie de 1.741 ha.

Los principales destinos de las exportaciones son EE.UU., con un $85 \%$ de participación, Holanda con un $14 \%$, Emiratos Árabes con un 0,6\% y el Reino Unido $0,4 \%$ de participación; estos envíos de flores de corte bordean los US\$ 3 millones ${ }^{2}$.

Debido al escaso nivel tecnológico con el que cuentan los pequeños productores, se ven excluidos de la posibilidad de exportar sus producciones y obligados a vender en sus propios predios o en el terminal de flores de Santiago, bajo la modalidad de consignación o venta directa, por lo que la rentabilidad de su actividad se ve desmejorada. Hoy en día se hace necesaria la búsqueda de nuevas alternativas para los productores de flores.

En esta búsqueda, Lisianthus (Eustoma grandiflorum) puede ser una de las especies que complemente la producción floral en determina-

Anónimo, 1999.

Anónimo, 2004 das épocas del año, correspondiendo esta a una especie muy atractiva para el consumidor, con gran variedad de colores y buena duración en florero $^{3}$. Su cultivo no presenta grandes problemas técnicos, las estructuras de soporte y conducción son semejantes a las utilizadas en producción de claveles, permitiendo a los productores diversificar los cultivos sin mayores complicaciones (Melgares de Aguilar, 1996).

Los tratamientos de postcosecha para Lisianthus aún no han sido muy estudiados, debido a la reciente introducción en Chile de esta especie, pero sí se han realizado estudios de postcosecha en otras especies florales entre las cuales destacan: rosas, claveles, crisantemos, en las que se utilizan distintas soluciones conservantes, compuestas principalmente por azúcar, hidroxiquinolina, nitrato de plata, citoquinina y ácido cítrico (Huber, 1994).

Con la finalidad de dar respuesta a los productores florales en relación a los tratamientos de postcosecha para Lisianthus, el presente trabajo plantea como hipótesis que el uso de retardadores de maduración permitiría postergar la apertura de botones florales de Lisianthus, una vez cosechados y almacenados en bodegas, utilizados por pequeños productores florales para estos fines.

\section{OBJETIVO GENERAL}

Evaluar la acción de dos soluciones conservantes en Lisianthus correspondientes a: Como®, aplicado en precosecha, y ácido cítrico en postcosecha, bajo condiciones de almacenamiento utilizadas por pequeños productores florales.

\section{OBJETIVOS ESPECÍFICOS}

Evaluar parámetros de floración, número de flores abiertas, número de flores senescentes, diámetro del botón y peso del mismo, con y sin modificadores de $\mathrm{pH}$ (ácido cítrico).

Evaluar el uso de Como® y su acción como retardante de floración en esta especie.

Evaluar la adición de ácido cítrico en soluciones preservantes de postcosecha para varas de Lisianthus.

3 Klasman, 2001. 


\section{MATERIAL Y MÉTODO}

\section{MATERIALES}

La investigación se llevó a cabo en la parcela "Agroaromas", ubicada en la localidad de "La Montaña de Teño", Teño, Séptima Región, entre los días 4 de abril y 1 de mayo del año 2003.

La Figura 1 muestra el material vegetal utilizado en la presente investigación, perteneciente al cultivar Heidi, el cual fue obtenido en la ciudad de La Serena, IV Región, siendo trasplantado, con fecha de octubre del año 2002, en la parcela "Agroaromas".

Las plántulas de Lisianthus fueron cultivadas en una nave existente en dicho predio, la cual tenía una ubicación este-oeste, utilizando para ello tres camellones de $1 \mathrm{~m}$ de ancho por $12 \mathrm{~m}$ de largo. Antes de trasplantar se procedió a desinfectar el suelo con Mancozeb 80\% PM (i.a.: Etilenbisditiocarbamato de manganeso más ión zinc).

\section{METODOLOGÍA}

La plantación se realizó a una distancia de $15 \mathrm{~cm}$ x $15 \mathrm{~cm}$, resultando así un total de 32 plantas $/ \mathrm{m}^{2}$. La fertilización durante el periodo de cultivo se realizó en base a Nitrofoska ${ }^{\circledR}$ foliar y nitrato de calcio, alternando entre un producto y otro una vez por semana.
A comienzos del mes de diciembre se procedió a aplicar ácido giberélico, una vez por semana, con el propósito de adelantar la floración.

Para el almacenaje de los botones se utilizó una bodega existente en el predio, a una distancia de $30 \mathrm{~m}$, en dirección norte de la nave, en la cual se cultivaban las plantas. Esta bodega presentaba la misma orientación de la plantación, es decir, este-oeste, y durante el estudio registró una temperatura promedio de $13{ }^{\circ} \mathrm{C}$ y una humedad relativa de $82 \%$.

Los botones florales fueron depositados en tarros de metal, los cuales presentaban una capacidad de $2 \mathrm{~L}$; en dichos contenedores se adicionó 1 L de agua y ácido cítrico a diferentes dosificaciones.

Los conservantes utilizados en el ensayo fueron los siguientes:

- Como® en una solución de $1 \mathrm{~cm}^{3} / \mathrm{L}$, aplicado en precosecha, específicamente 12 días antes de cosecha. Como® corresponde a un fertilizante líquido, compuesto por sales de cobalto y molibdeno, el cual aplicado a frutales y flores antes de cosecha retrasa el fenómeno de senescencia de los tejidos.

- Ácido cítrico en solución de 0,3 mg/L, aportado en postcosecha, cuya acción corresponde a bajar el pH, con lo cual disminuye la presencia de flora microbiana en el agua utilizada para conservar las flores.

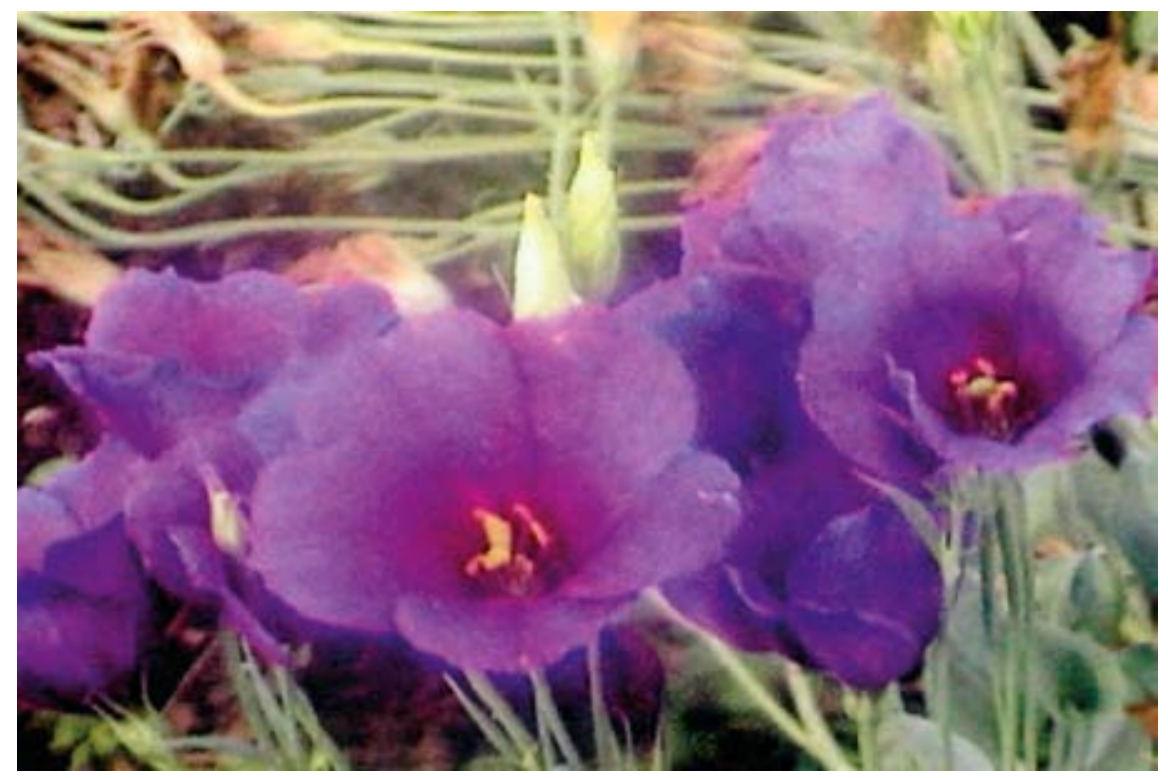

Figura 1. Material vegetal, cultivar Heidi, utilizado en los ensayos. 
Para la aplicación de Como® en las plantas se utilizó una bomba de espalda de $15 \mathrm{~L}$ de marca Solo, calibrada para aplicar $0,5 \mathrm{~L} / \mathrm{min}$.

Los materiales utilizados para la obtención del peso y diámetro de la vara fueron una pesa digital y un pie de metro.

Se evaluó la aplicación de ácido cítrico en tratamiento de postcosecha, para plantas tratadas y no tratadas en precosecha, con el producto comercial denominado Como®.

\section{TRATAMIENTOS}

To: Agua postcosecha.

$\mathrm{T}_{1}$ : Aplicación de Como® en precosecha, más agua postcosecha.

$\mathrm{T}_{2}$ : Aplicación de Como® en precosecha, más $0,2 \mathrm{~mL}$ ácido cítrico/L de agua en postcosecha.

$\mathrm{T}_{3}$ : Aplicación de Como® en precosecha, más $0,3 \mathrm{~mL}$ ácido cítrico/L de agua en postcosecha.

$\mathrm{T}_{4}$ : Aplicación de Como® en precosecha, más $0,5 \mathrm{~mL}$ ácido cítrico/L de agua en postcosecha.

$\mathrm{T}_{5}:$ 0,2 $\mathrm{mL}$ ácido cítrico/L de agua en postcosecha. $\mathrm{T}_{6}: 0,3 \mathrm{~mL}$ ácido cítrico/L de agua en postcosecha. $\mathrm{T}_{7}: 0,5 \mathrm{~mL}$ ácido cítrico/L de agua en postcosecha.

El día 4 de abril a las 15 horas se realizó una elección de varas completamente al azar, selec- cionando un camellón de $1 \mathrm{~m}$ de ancho por $12 \mathrm{~m}$ de largo, el cual contaba con varas en similares condiciones, esto es, con botones comenzando a tomar color, para luego aplicar una dosis de $4 \mathrm{~L}$ de Como® en una concentración de $1 \mathrm{~cm}^{3} / \mathrm{L}$ en todo el camellón, operación que tomó un tiempo de 8 minutos.

Posteriormente, el día 16 de abril, doce días después de aplicado el conservante de precosecha, se procedió a cortar 192 varas, que habían sido tratadas con Como® y otras 192 varas no tratadas con dicho producto, las que presentaban de dos a tres botones florales abiertos, luego fueron depositadas en depósitos que contenían un litro de agua con soluciones de ácido cítrico, en concentraciones de $(0,2-0,3-0,5) \mathrm{mL} / \mathrm{L}$ de agua, y conservados dentro de la bodega de almacenaje, la cual contaba con luz eléctrica, simulando de esta forma las condiciones de almacenaje utilizadas por pequeños productores de flores.

Los contenedores utilizados para el almacenamiento fueron debidamente desinfectados con una solución de cloro al $1 \%$ y rotulados con la fecha de inicio del experimento y la solución contenida en cada uno de ellos, según se muestra en la Figura 2.

Durante el ensayo se evaluaron cuatro parámetros de calidad en flores: peso de la vara $(\mathrm{g})$, diámetro del botón $(\mathrm{cm})$, número de flores abier-

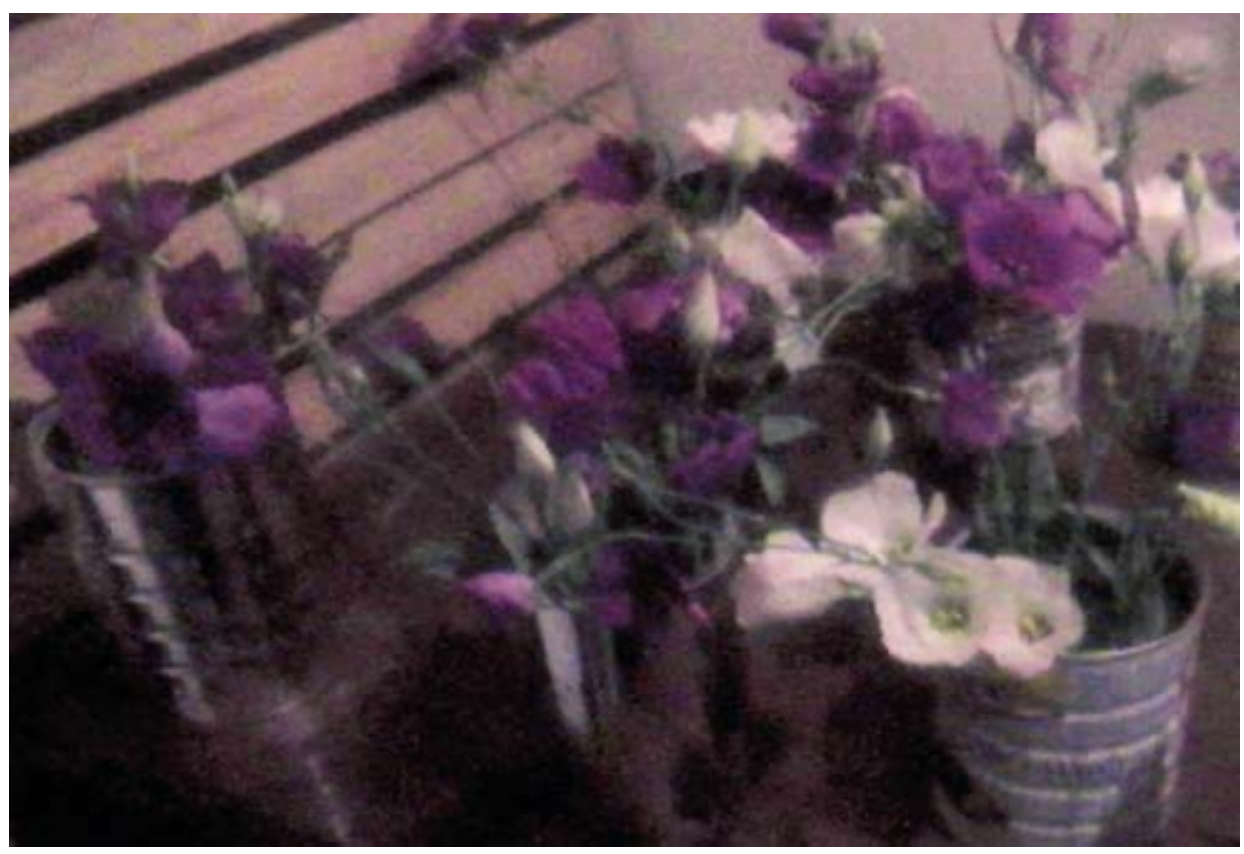

Figura 2. Varas depositadas dentro de los contenedores, en la bodega de almacenamiento, a $13{ }^{\circ} \mathrm{C}$ y $82 \%$ de H.R. 
tas y número de flores senescentes; para realizar dichas mediciones se evaluó en cada vara sólo el botón floral apical. Las mediciones se efectuaron a los 0-5-10 y 15 días de almacenadas las varas, realizándose la primera de estas el día 16 de abril y la última el día 1 de mayo. Para cada medición se analizó un total de tres botones por los siete tratamientos, lo que totaliza una cantidad de 84 botones $(4 \times 3 \times 7=84)$.

Las mediciones tomadas durante el ensayo fueron realizadas dentro de la misma bodega de almacenamiento.

En el Cuadro 1 se indican los parámetros de calidad medidos por tratamiento durante el ensayo.

\section{DISEÑO Y ANÁLISIS ESTADÍSTICO}

El diseño experimental utilizado correspondió a bloques completos al azar, con siete tratamientos y tres repeticiones, utilizando el producto Como ${ }^{\circledR}$ en precosecha más ácido cítrico en postcosecha, o sólo tratadas con ácido cítrico en postcosecha. El factor de variación para los bloques correspondió a los días transcurridos después de cosechadas las varas.

Se evaluaron los cuatro parámetros de calidad anteriormente señalados, en evaluaciones periódicas cada cinco días, desde el ingreso a la bodega de almacenamiento $\left(\mathrm{t}_{0}\right)$ hasta completar 15 días de permanencia $\left(t_{15}\right)$.

La unidad experimental correspondió a 48 varas florales contenidas en un contenedor de metal con una solución preservante, mientras que el testigo $\left(\mathrm{T}_{0}\right)$ sólo se evaluó con agua pura.

Los resultados promedio de las repeticiones por el tratamiento y parámetro, medidos desde $\mathrm{t}_{0}$ hasta $t_{15}$ fueron cotejados por el test de Tuckey $(p<0,05)$.

\section{RESULTADOS Y DISCUSIÓN}

\section{NÚMERO DE FLORES ABIERTAS}

Durante la permanencia de las varas florales en la bodega de almacenamiento fue posible observar un mayor número de flores abiertas, desde $\mathrm{t}_{0}$ hasta $\mathrm{t}_{15}$, con los tratamientos $\mathrm{T}_{2} \mathrm{y} \mathrm{T}_{6}$, aunque estos resultados no se ven reflejados en el cuadro, puesto que según el test de Tuckey al 5\% de probabilidad no existe diferencia significativa.

En una vara de Lisianthus se esperaría que todos los botones florales que se encuentran empezando a tomar color al momento de ser cosechados abrieran, por lo que en el presente ensayo se esperaba encontrar abiertas entre 4 y 5 flores. El bajo número

Cuadro 1

Parámetros medidos por tratamiento $(T)$, desde $t_{0}$ hasta $t_{15}$

\begin{tabular}{|c|c|c|c|c|}
\hline \multirow{3}{*}{ (T) Tratamientos } & Diámetro del botón & Peso del botón & $\mathbf{N}^{0}$ flores del botón & $\begin{array}{c}\mathbf{N}^{\mathrm{o}} \text { flores } \\
\text { abiertas senescentes }\end{array}$ \\
\hline & $\begin{array}{l}\text { Días después } \\
\text { de cosecha }\end{array}$ & $\begin{array}{c}\text { Días después } \\
\text { de cosecha }\end{array}$ & $\begin{array}{l}\text { Días después } \\
\text { de cosecha }\end{array}$ & $\begin{array}{l}\text { Días después } \\
\text { de cosecha }\end{array}$ \\
\hline & $\begin{array}{lllll}\text { (t) } & 0 & 5 & 10 & 15\end{array}$ & $\begin{array}{llll}0 & 5 & 10 & 15\end{array}$ & $\begin{array}{llll}0 & 5 & 10 & 15\end{array}$ & $\begin{array}{llll}0 & 5 & 10 & 15\end{array}$ \\
\hline $\mathrm{T}_{0}$ & & & & \\
\hline $\mathrm{T}_{1}$ & & & & \\
\hline $\mathrm{T}_{2}$ & & & & \\
\hline $\mathrm{T}_{3}$ & & & & \\
\hline $\mathrm{T}_{4}$ & & & & \\
\hline $\mathrm{T}_{5}$ & & & & \\
\hline $\mathrm{T}_{6}$ & & & & \\
\hline $\mathrm{T}_{7}$ & & & & \\
\hline
\end{tabular}


de flores abiertas durante la vida en postcosecha de Lisianthus, observado en el Cuadro 2, puede ser explicado por la ausencia de azúcar suplementario, el cual es fundamental para este efecto. Chahín et al. (2002) señalan al respecto que almidones y diferentes formas de azúcares almacenados en tallos, hojas y pétalos proveen la energía necesaria para que la flor complete su desarrollo.

El azúcar retarda la senescencia, mejora el balance hídrico y el potencial osmótico, además provee de un sustrato disponible para la respiración, es decir, aporta energía a los tejidos de los pétalos. Lo anterior mantiene un alto peso fresco en el tallo, induciendo al cierre de los estomas en hojas, y con esto reduce la pérdida de agua. Además, el azúcar ayuda a mantener la integridad, estructura y función de las membranas (Halevy y Mayak, 1981).

Han (2003) señala a la sacarosa como el más abundante fotosintato proveniente del carbono, la cual es fuente fundamental para el desarrollo de los pétalos en flores; por otra parte, Huang y Chen (2002) señalan que la aplicación de citoquinina exógena retrasaría el fenómeno de la senescencia ayudando al traslado de solutos desde las partes más viejas de la planta hacia los tejidos en formación.

Al respecto, Huber (1994) indica que existe un movimiento del azúcar en la planta provocado por la transpiración, lo cual lleva a una acumulación de

Cuadro 2

Número de flores abiertas promedio, por tratamiento, a los 0-5-10 y 15 días de cosechadas las varas. Valores promedio de tres repeticiones

\begin{tabular}{|c|c|c|c|c|}
\hline \multirow{2}{*}{ Tratamiento } & \multicolumn{4}{|c|}{ Días } \\
\cline { 2 - 5 } & Día 0 & Día 5 & Día 10 & Día 15 \\
\hline $\mathrm{T}_{0}$ & 2,0 & 2,7 & 3,0 & 3,0 \\
\hline $\mathrm{T}_{1}$ & 2,3 & 2,7 & 2,7 & 3,0 \\
\hline $\mathrm{T}_{2}$ & 2,7 & 3,0 & 2,7 & 3,0 \\
\hline $\mathrm{T}_{3}$ & 2,0 & 3,0 & 3,0 & 3,0 \\
\hline $\mathrm{T}_{4}$ & 1,0 & 2,3 & 3,0 & 3,0 \\
\hline $\mathrm{T}_{5}$ & 1,7 & 2,7 & 3,0 & 3,0 \\
\hline $\mathrm{T}_{6}$ & 2,7 & 3,0 & 3,0 & 3,0 \\
\hline $\mathrm{T}_{7}$ & 2,7 & 2,3 & 2,7 & 3,0 \\
\hline
\end{tabular}

azúcar en los tallos y hojas, para luego ser trasladado a los pétalos vía floema. Durante la transferencia de azúcar, desde el xilema al floema, ocurre una inversión catalizada por la enzima invertasa, la cual transforma la sacarosa en glucosa y fructosa. Una vez alcanzado el floema, los azúcares reducidos son utilizados para el metabolismo o bien, reconvertidos en sacarosa, la cual puede ser traslocada hacia otras áreas.

Por otra parte, la hora en que se realizó la cosecha de las varas, 3 p.m., influyó negativamente puesto que según lo señalado por Chahín et al. (2002) el tiempo más apropiado para realizar esta labor es temprano por la mañana, ya que las plantas se encuentran totalmente turgentes, siendo el contenido de agua en las flores el factor de mayor relevancia en la vida de postcosecha. Debido al fenómeno de marchitamiento del material, puede resultar una disminución de la longevidad de la flor, por lo que se recomienda además regar antes de la cosecha para permitir una mejor hidratación de las flores y, aunque para el presente ensayo se realizó dicha labor, la hora de cosecha señalada provocó un efecto negativo, ya que la tasa respiratoria del material vegetal, a la hora que se realizó el corte de las varas, debe haber sido alta, provocando un estrés en las mismas.

Otro factor importante a considerar es que las flores cortadas constituyen órganos inmaduros, que tienen una alta relación superficie/volumen, por lo que su actividad metabólica es alta y pierden muy rápidamente agua. Por este motivo es que es recomendable almacenar las flores de corte a la temperatura más baja y a la humedad relativa más alta que puedan tolerar (Chahín et al., 2002). En el presente ensayo se buscó llevar a cabo los experimentos según la situación propia de los pequeños agricultores florícolas de la provincia, quienes no cuentan con bodegas acondicionadas para brindar la temperatura y humedad relativa sugeridas por la literatura y propicias para Lisianthus.

\section{NÚMERO DE FLORES SENESCENTES}

Transcurridos 10 días de almacenadas las varas en la bodega, se observó una variación entre los tratamientos efectuados con Como ${ }^{\circledR}$ en precosecha $\left(\mathrm{T}_{1}, \mathrm{~T}_{2}, \mathrm{~T}_{3}\right.$ y $\left.\mathrm{T}_{4}\right)$ y los tratamientos sin dicho producto $\left(\mathrm{T}_{5}, \mathrm{~T}_{6} \mathrm{y} \mathrm{T}_{7}\right)$, encontrándose un menor número de flores senescentes en los tratamientos con aplicación Como ${ }^{\circledR}$ en precosecha, lo que no se 
reflejó según el análisis estadístico (Cuadro 3). La menor cantidad de flores senescentes, observada durante el almacenaje en la bodega, para las varas con aplicación de Como® en precosecha, se puede explicar por una acción sinérgica entre el producto Como® y el ácido cítrico, lo que pudo haber permitido mantener en mejor forma el parámetro medido y así aparentemente prolongar la vida de postcosecha en flores de Lisianthus, considerando que el producto adicionado en precosecha llamado Como® inhibe la producción de etileno en los órganos vegetales.

El etileno corresponde a la "hormona de la maduración", la que en su estado natural es un gas, producto normal del proceso respiratorio de las plantas y que actúa a concentraciones muy bajas. Su síntesis comienza con el aminoácido metionina, vía una ruta de la que forman parte, como productos intermedios, la S-adenosil-metionina (SAM) y el ácido 1-aminociclopropano-l-carboxílico (ACC). La conversión de SAM en ACC, por la enzima ACC sintetasa, parece ser la etapa limitante de la velocidad de biosíntesis de etileno. Sin embargo, estudios recientes han demostrado que se necesita otra enzima, la enzima formadora de etileno, para convertir la ACC en etileno; esta enzima corresponde a la ACC oxidasa, la cual es una enzima que está asociada a la membrana, es lábil, sensible al oxígeno y se inactiva fácilmen-

Cuadro 3

Número de flores senescentes promedio, por tratamiento, a los 0-5-10 y 15 días de cosechadas las varas. Valores promedio de tres repeticiones

\begin{tabular}{|c|c|c|c|c|}
\hline \multirow{2}{*}{ Tratamiento } & \multicolumn{4}{|c|}{ Días } \\
\cline { 2 - 5 } & Día 0 & Día 5 & Día 10 & Día 15 \\
\hline To & 0 & 2,7 & 3,0 & 3,0 \\
\hline $\mathrm{T}_{1}$ & 0 & 0,3 & 2,0 & 3,0 \\
\hline $\mathrm{T}_{2}$ & 0 & 1,7 & 1,7 & 3,0 \\
\hline $\mathrm{T}_{3}$ & 0 & 0,3 & 2,0 & 3,0 \\
\hline $\mathrm{T}_{4}$ & 0 & 1,0 & 0,7 & 2,7 \\
\hline $\mathrm{T}_{5}$ & 0 & 1,7 & 2,0 & 3,0 \\
\hline $\mathrm{T}_{6}$ & 0 & 2,7 & 2,7 & 3,0 \\
\hline $\mathrm{T}_{7}$ & 0 & 2,3 & 3,0 & 3,0 \\
\hline
\end{tabular}

te. La ACC oxidasa es inhibida por la anaerobiosis, por temperaturas superiores a los $35^{\circ} \mathrm{C}$ y por los iones cobalto. Por lo tanto, el cobalto presente en la formulación de Como® actúa como un antagonista, inhibiendo la transformación de ácido 1-aminociclopropano-1-carboxilo (ACC) a etileno (Barceló, 2001). El etileno en flores es el responsable de la senescencia y abscisión de pétalos y botones, acción que contribuye al equilibrio del botón floral, pues permite que los botones no abiertos reciban los fotosintatos necesarios para abrir, es por esto que para que botones de la vara floral abran, otros deben absicionar. El mecanismo mediante el cual los pétalos abscisionan corresponde a aquel fenómeno en que los pétalos se vuelven completamente sensibles al etileno, responden con una producción explosiva y autocatalítica de la hormona, lo que causa un marchitamiento de los pétalos en respuesta a una mayor permeabilidad de la membrana plasmática y el tonoplasto, siguiendo con la pérdida de solutos y luego del agua a las paredes celulares y probablemente a los espacios intercelulares (Salisbury y Ross, 1991).

Además de esta acción inhibitoria de etileno, el producto $C o m o \circledR$ tendría por característica el aumento de la tasa fotosintética del material vegetal en cuestión y con dicho aumento en la fotosíntesis se produciría un aumento en los fotosintatos disponibles al momento de la cosecha y posterior postcosecha; del mismo modo, el ácido cítrico actuaría permitiendo que estos fotosintatos, representados por azúcares reductores, no sufrieran alteraciones en sus radicales libres y así estar disponibles para las varas de Lisianthus en postcosecha. Autores como Melgares de Aguilar (1996) han señalado que el efecto del etileno es menor sobre la senescencia de las flores de Lisianthus y los tratamientos para inhibir la acción de esta hormona no presentarían mayores beneficios. En los tratamientos $\mathrm{T}_{2}$ (aplicación de Como ${ }^{\circledR}$ en precosecha, más $0,2 \mathrm{~mL}$ ácido cítrico/ $\mathrm{L}$ de agua en postcosecha) y $\mathrm{T}_{5}(0,2 \mathrm{~mL}$ ácido cítrico/L de agua en postcosecha), durante las mediciones dentro de la bodega, se pudo apreciar que no existió ninguna diferencia entre las varas tratadas con el producto Como ${ }^{\circledR}$ en precosecha y las varas sin dicho tratamiento. Esta similitud entre los resultados podría indicar que la dosis de $0,2 \mathrm{~mL}$ de ácido cítrico por cada $L$ de agua no sería suficiente para realizar un eficiente control de los microorganismos en el agua utilizada para el almacenamiento de las varas de Lisianthus. El control que el ácido cítrico ejerce sobre 
los microorganismos presentes en el agua, utilizada para el almacenamiento de las varas de Lisianthus, está dado por la reducción de $\mathrm{pH}$ en dicho medio y con esto afectaría directamente a las bacterias que estuvieran presentes en ella, puesto que los cambios bruscos en el $\mathrm{pH}$ del medio circundante en el que se desarrollan los microorganismos pueden resultar letales para ellos, debido a que afectan su membrana plasmática, causando cambios en la homeostasis celular. Chahín et al. (2002) señalan que los microorganismos que se desarrollan en agua utilizada para el almacenamiento impedirían el paso de la misma a través del xilema, debido a que se forma un tapón mucoso, el cual provoca la oclusión de los vasos conductores. Al respecto, Huber (1994) señaló que también se genera una oclusión de los vasos conductores, debido a sustancias gomosas, pectinas o carbohidratos provenientes de la rotura de las paredes celulares. Se señala además que los fenoles producto de la degradación de proteínas reaccionan con otros compuestos formando sustancias leñosas de carácter insoluble, como lo es la lignina, siendo todos estos compuestos los que contribuirían a obstruir los haces conductores.

\section{DIÁMETRO DEL BOTÓN}

Para el parámetro de calidad, diámetro del botón en postcosecha, sí se observaron diferencias significativas entre los tratamientos $\mathrm{T}_{4} \mathrm{y} \mathrm{T}_{6}$, según Tuckey al $5 \%$ de probabilidad, resultando ser el tratamiento $\mathrm{T}_{6}(0,3 \mathrm{~mL}$ ácido cítrico/ $\mathrm{L}$ de agua en postcosecha) el que presentó los menores diámetros durante la vida de postcosecha, y que resultó significativamente distinto de $\mathrm{T}_{4}\left(\mathrm{Como}{ }^{\circledR}\right.$ en precosecha, más $0,5 \mathrm{~mL}$ de ácido cítrico/L de agua, en postcosecha), el tratamiento con los mayores diámetros

El tratamiento $\mathrm{T}_{6}$ no tuvo un buen efecto sobre la calidad de las varas en postcosecha, expresada con el parámetro de calidad diámetro del botón, puesto que presentó diámetros significativamente menores $\mathrm{T}_{4}$, el cual contaba con aplicación de Como® en precosecha, lo que indica que el diámetro del botón, según se observa en el Cuadro 4 , se ve favorecido con la aplicación de Como ${ }^{\circledR}$ en precosecha, el cual proveería de fotosintatos disponibles para las varas en postcosecha, siendo estos los que ayudarían a favorecer el cierre estomático, disminuyendo así las pérdidas de agua por transpiración y simultáneamente respiración. Dicho proceso ayudaría a que el botón continuara

\section{CUADRO 4}

Diámetro del botón (cm) promedio, por tratamiento, a los 0-5-10 y 15 días de cosechadas las varas. Valores promedio de tres repeticiones

\begin{tabular}{|c|c|c|c|c|}
\hline Tratamiento & Día 0 & Día 5 & Día 10 & Día 15 \\
\hline $\mathrm{T}_{0}$ & $52,7 \mathrm{ab}$ & $57,0 \mathrm{ab}$ & $8,0 \mathrm{c}$ & $5,0 \mathrm{ab}$ \\
\hline $\mathrm{T}_{1}$ & $53,0 \mathrm{ab}$ & $53,3 \mathrm{ab}$ & $40,3 \mathrm{ab}$ & $5,0 \mathrm{ab}$ \\
\hline $\mathrm{T}_{2}$ & $38,0 \mathrm{~b}$ & $48,3 \mathrm{~b}$ & $27,7 \mathrm{~b}$ & $5,0 \mathrm{ab}$ \\
\hline $\mathrm{T}_{3}$ & $57,3 \mathrm{ab}$ & $44,0 \mathrm{~b}$ & $29,0 \mathrm{~b}$ & $5,0 \mathrm{ab}$ \\
\hline $\mathrm{T}_{4}$ & $64,7 \mathrm{a}$ & $64,7 \mathrm{a}$ & $51,0 \mathrm{a}$ & $5,0 \mathrm{ab}$ \\
\hline $\mathrm{T}_{5}$ & $54,3 \mathrm{ab}$ & $54,3 \mathrm{ab}$ & $19,7 \mathrm{~b}$ & $5,0 \mathrm{ab}$ \\
\hline $\mathrm{T}_{6}$ & $39,0 \mathrm{~b}$ & $8,0 \mathrm{c}$ & $5,0 \mathrm{c}$ & $5,0 \mathrm{ab}$ \\
\hline $\mathrm{T}_{7}$ & $46,7 \mathrm{~b}$ & $59,0 \mathrm{ab}$ & $5,0 \mathrm{c}$ & $5,0 \mathrm{ab}$ \\
\hline
\end{tabular}

Valores con igual letra dentro de cada columna, no difieren estadísticamente, según Tuckey a un nivel de protección del 5\%.

con su apertura, aun después de cosechada la vara (Barceló et al., 2001).

El estado de completa apertura del botón floral sería para el caso de las flores un indicador de la madurez comercial, la cual en sí es difícil determinar, ya que corresponden a atributos subjetivos y específicos, dependientes de los gustos de los consumidores y del segmento al cual va dirigido el producto. En términos generales, el tamaño y la forma del botón o la vara floral corresponden a un índice importante de la madurez comercial en los productos ornamentales (Wills et al., 1999).

Otro aspecto importante a considerar en la vida de postcosecha, y que contribuye a la calidad tanto del botón como de la vara floral, corresponde al color. Las clorofilas constituyen el pigmento fundamental de las hojas verdes. Los responsables del color violeta que presentaron las flores del cultivar Heidi, utilizados en el presente ensayo son los antocianos, los cuales se encuentran en solución en las vacuolas. Sin embargo, los colores observados son muy dependientes del $\mathrm{pH}$ de la savia de la flor (Wills et al., 1999).

Cabe destacar que durante la vida de postcosecha en Lisianthus se observó un leve ennegrecimiento del botón, debido a que los antocianos a valores de $\mathrm{pH}$ superiores a siete tienden a adquirir tonalidades 
más oscuras. Dicho fenómeno se debe al agotamiento de los azúcares como sustrato respirable y a la transición metabólica hacia el catabolismo de las proteínas, que libera grupos amina y desplaza el pH hacia valores más alcalinos, siendo estos cambios propios de la senescencia de los pétalos (Gil, 2001; Han, 2003).

\section{PESO DEL BOTÓN}

El Cuadro 5 deja ver que existieron diferencias significativas según el test de Tuckey al 5\%, diferenciándose el tratamiento $\mathrm{T}_{4}$ (aplicación de Como ${ }^{\circledR}$ en precosecha, más $0,5 \mathrm{~mL}$ ácido cítrico/L de agua en postcosecha) de los tratamientos $\mathrm{T}_{6}$ ( $0,3 \mathrm{~mL}$ ácido cítrico/L de agua en postcosecha) y $\mathrm{T}_{7}(0,5 \mathrm{~mL}$ ácido cítrico/ $\mathrm{L}$ de agua en postcosecha), estos últimos resultan ser iguales entre ellos.

Plantas tratadas con el tratamiento $\mathrm{T}_{4}$ presentaron mayores pesos de botones, respecto de aquellas tratadas con los tratamientos $\mathrm{T}_{6} \mathrm{y}_{7}$, lo que indicaría que se produjo una interacción entre el producto Como ${ }^{\circledR}$ aplicado en precosecha y ácido cítrico aplicado en postcosecha, ayudando a que la flor mejorara la absorción de agua y por tanto se vería reflejado en el peso del botón.

Huber (1994), trabajando con gladiolos, indicó que las flores una vez cortadas y puestas en agua

\section{Cuadro 5}

Peso del botón (g) promedio, por tratamiento, a los 0-5-10 y 15 días de cosechadas las varas. Valores promedio de tres repeticiones

\begin{tabular}{|c|l|l|l|l|}
\hline Tratamiento & Día 0 & Día 5 & Día 10 & Día 15 \\
\hline $\mathrm{T}_{0}$ & $7,0 \mathrm{ab}$ & $7,67 \mathrm{ab}$ & $3,0 \mathrm{c}$ & $2,67 \mathrm{c}$ \\
\hline $\mathrm{T}_{1}$ & $4,33 \mathrm{~b}$ & $5,67 \mathrm{~b}$ & $4,0 \mathrm{~b}$ & $3,67 \mathrm{~b}$ \\
\hline $\mathrm{T}_{2}$ & $6,67 \mathrm{ab}$ & $8,0 \mathrm{a}$ & $5,67 \mathrm{ab}$ & $4,67 \mathrm{~b}$ \\
\hline $\mathrm{T}_{3}$ & $7,67 \mathrm{a}$ & $7,0 \mathrm{ab}$ & $3,0 \mathrm{c}$ & $2,33 \mathrm{c}$ \\
\hline $\mathrm{T}_{4}$ & $6,0 \mathrm{ab}$ & $8,67 \mathrm{a}$ & $7,67 \mathrm{a}$ & $7,0 \mathrm{a}$ \\
\hline $\mathrm{T}_{5}$ & $4,33 \mathrm{~b}$ & $5,0 \mathrm{~b}$ & $3,67 \mathrm{~b}$ & $5,67 \mathrm{ab}$ \\
\hline $\mathrm{T}_{6}$ & $4,33 \mathrm{~b}$ & $5,33 \mathrm{~b}$ & $3,33 \mathrm{c}$ & $2,67 \mathrm{c}$ \\
\hline $\mathrm{T}_{7}$ & $6,67 \mathrm{ab}$ & $3,0 \mathrm{c}$ & $3,0 \mathrm{c}$ & $2,33 \mathrm{c}$ \\
\hline
\end{tabular}

Valores con igual letra dentro de cada columna, no difieren estadísticamente, según Tuckey a un nivel de protección del 5\%. inicialmente aumentaron su peso y luego éste decreció; lo anterior se debería a que el corte de la vara desde la planta se realizó en seco, lo que ocasionó un déficit de agua en las células de las plantas a nivel de tallo, y por ende disminuyó el potencial hídrico en los tejidos, creando un efecto de capilaridad, lo que una vez ubicadas las varas en el florero favorecería la absorción de agua. Sin embargo, al aumentar el contenido de agua en los tejidos, al inicio de la vida en florero, el potencial disminuye y por tanto el gradiente de presión es menor, por lo que el consumo de agua de la vara va decreciendo. No obstante, las flores de corte suelen proveerse de agua durante parte o todo el período posproducción (Wills et al., 1999).

La turgencia es el parámetro de frescura más relevante en flores de corte, esta corresponde al resultado del balance entre el agua absorbida y la pérdida de la misma. La flor se ve impedida para absorber agua por la oclusión de vasos conductores, lo cual se debería a sustancias gomosas, pectinas o carbohidratos en su naturaleza, provenientes de la rotura de paredes celulares y a la presencia de microorganismos en el agua (Huber, 1994).

Chain et al. (2002) señalan que el pH del agua es un factor importante, recomendándose valores entre 3 y 4 , dado que esto mejora el control microbiano y junto con esto se favorece el ascenso del agua por el xilema.

A pesar de las condiciones de la bodega de almacenamiento utilizada para el presente ensayo, la cual no contaba con sistemas de control para la temperatura, ni para humedad relativa, ya que se pretendía simular las condiciones de manejo de postcosecha que utilizan los pequeños productores de flores de la provincia, fue factible prolongar la vida de postcosecha en varas de Lisianthus, entendida ésta según Chaín et al. (2002) como el período de tiempo en el cual la flor se mantiene atractiva visualmente, conservando su forma y color, según la especie y cultivar, sumado al follaje acompañante que se observó sano, completo y brillante.

La mantención de la calidad de varas de Lisianthus en postcosecha, según lo observado dentro de la bodega de almacenaje, se hizo posible utilizando los productos químicos Como ${ }^{\circledR}$ y ácido cítrico, lo que permitió mejorar el diámetro de los botones florales y el peso de estos en todo el período del ensayo, considerado satisfactorio para fines comerciales. 


\section{CONCLUSIONES}

La aplicación del producto Como® en precosecha presentó un efecto positivo en la calidad de la vara de Lisianthus en postcosecha.

Los tratamientos $\mathrm{T}_{5}, \mathrm{~T}_{6} \mathrm{y} \mathrm{T}_{7}$, en los que sólo se adicionó ácido cítrico en postcosecha, no resultaron ser eficientes en aumentar el número de flores abiertas, disminuir el número de flores senescentes y aumentar el diámetro y peso del botón.

\section{LITERATURA CITADA}

ANÓNIMO. 1999. Boletín trimestral FIA, № 1 , octubre de 1999. Disponible en www.fia.cl/difus/boletin/bflor/bfl 099. htm. Conectada el 12 de julio 2004.

ANÓNIMO. 2004. La Segunda. Disponible en www.bayercropscience.cl/noticias/vernoticia.asp?id=434. Conectada el 12 de julio 2004.

BARCELÓ, J., NICOLÁS, G., SABATER, B. Y SÁNCHEZ, R. 2001. Fisiología Vegetal. $7^{a}$ edición. Ediciones Pirámide. $566 \mathrm{p}$.

CHAHÍN, M., MONTESINOS,A.Y VERDUGO, G. 2002. Manejo de postcosecha de flores. Boletín INIA N ${ }^{\circ} 82$. Temuco, Chile.

GIL, G. 2001. Madurez de la fruta y manejo postcosecha. $1^{\mathrm{a}}$ edición. Ediciones Salesianos.

HALEVY, A. AND MAYAK, S. 1981. Senescence and postharvest physiology of cut flowers II. Horticultural Reviews 1 (5): 204-236.

HAN, S. 2003. Role of sugar in the vase solution on postharvest flower and leaf quality of oriental lily "stargazer". HortSciencie 38 (3): 412-416.
Los tratamientos $\mathrm{T}_{\mathrm{I}}, \mathrm{T}_{2}, \mathrm{~T}_{3} \mathrm{y} \mathrm{T}_{4}$ en los que se realizó aplicación de Como® en precosecha no resultaron ser eficientes en retardar la floración en varas de Lisianthus.

La aplicación del producto Como® en precosecha, más ácido cítrico en postcosecha, resultó ser un eficiente tratamiento en varas de Lisianthus de corte, bajo similares condiciones de almacenamiento a las utilizadas por pequeños productores florales de la provincia de Curicó, esto representado en el aumento del diámetro y peso del botón.

HUANG, K. AND CHEN, W. 2002. Bensil Adenina and sucrose increase vase Ufe of cut Eustoma flowers. HortSciencie 37 (3): 547-549.

HUBER, C. 1994. Estudio de tratamientos de conservación de postcosecha de flores de gladiolo (Gladiolus hybridus). Tesis Lie. Agr. Valdivia, Universidad Austral de Chile, Facultad de Ciencias Agrarias. $121 \mathrm{p}$.

KLASMAN, R. 2001. Enfriar las flores. Disponible en www. buscagro.com/biblioteca/RolandoKlasman.pdf. Conectada el 20 de agosto de 2003.

MELGARES DE AGUILAR, J. 1996. El cultivo de Lisianthus I parte. Horticultura 113: 13-16.

SALISBURY, F. Y ROSS, C. 1991. Fisiología vegetal. $4^{\text {a }}$ edición. Editorial Iberoamérica.

WILLS, R., MCGLASSON, B., GRAHAM, D. AND JOYCE, D. 1999. Introducción a la fisiología y manejo postcosecha de frutas, hortalizas y plantas ornamentales. $2^{\text {a }}$ edición. Editorial Acribia. 